(2) Open Access Full Text Article

REVIEW

\title{
A novel lidocaine hydrochloride ophthalmic gel for topical ocular anesthesia
}

This article was published in the following Dove Press journal:

Local and Regional Anesthesia

5 August 2010

Number of times this article has been viewed

\section{HR Shah' \\ E Reichel $^{2}$ \\ BG Busbee ${ }^{3}$}

'New England Eye Center Boston, MA, USA; ${ }^{2}$ Vitreoretinal Service, New England Eye Center/Tufts University School of Medicine, Boston, MA, USA;

${ }^{3}$ Tennessee Retina, Nashville, TN, USA
Correspondence: Brandon G Busbee Tennessee Retina, 345 23rd Ave N, Suite 350, Nashville, TN 37203, USA Email bgbusbee@yahoo.com

\begin{abstract}
Topical anesthetics play an important role in the practice of ophthalmology, both for procedures in the office and in the operating room. The need for safe, long-acting topical ocular anesthetic agents is ongoing, and has been highlighted by the increase of intravitreal administration of pharmacologic agents. Current practices for ocular anesthesia include subconjunctival injection of $2 \%$ aqueous lidocaine, topical $2 \%$ lidocaine drops and topical $0.5 \%$ tetracaine. Tetracaine is not yet FDA approved, and is associated with corneal epithelial toxicity and delayed epithelial healing after multiple administrations. Lidocaine jelly ( $2 \%)$ preparations have been reported to be beneficial in several systemic procedures, including those of the upper airway, dental, urogenital, and gastrointestinal. It has been theorized, and recent studies support the idea, that gel formulations of lidocaine may enhance anesthetic effect, and therefore be superior to anesthetic solutions for topical cataract surgery. The viscous nature of gel formulations is thought to lengthen contact time, resulting in better anesthesia at lower drug concentrations. Furthermore, several studies suggest that lidocaine is bactericidal and bacteriostatic, and may have a supplementary role in preventing and treating surgical site infections. Akten ${ }^{\mathrm{TM}}$, lidocaine 3.5\% gel (Akorn, Buffalo Grove, IIlinois) was FDA approved for all ophthalmic procedures in October 2008. This gel is a preservative-free, lidocaine-based anesthetic gel consisting of $35 \mathrm{mg} / \mathrm{mL}$ of lidocaine hydrochloride. We describe the properties, including chemical structure, indications, evidence of support, use, adverse effects, and precautions, which we believe enable Akten to provide superior anesthesia, while minimizing side effects.
\end{abstract}

Keywords: Akten, lidocaine gel, topical anesthetic, ocular surgery

\section{Introduction/background}

Topical anesthetics play an important role in the practice of ophthalmology, both for procedures in the office and in the operating room. Until October 2008, the only Food and Drug Administration (FDA)-approved topical anesthetic preparation for ocular procedures was proparacaine. It is only available in solution form. Furthermore, its drop formulation typically requires repeated applications to achieve adequate surface anesthesia and often adjunctive intracameral injection of lidocaine solution is necessary for achieving adequate anesthesia for intraocular procedures. Tetracaine is another topical anesthetic frequently used for topical anesthesia in ophthalmology. It is not FDA-approved, and is known to be associated with corneal epithelial toxicity and delayed epithelial healing after multiple administrations. Topical ophthalmic anesthetic preparations are typically acidic, which contributes to the stinging sensation when first applied.

Ocular anesthesia can be delivered by a variety of routes. This includes retrobulbar and peribulbar injection, sub-Tenon's injection, subconjunctival injection, intracameral 
injection and topical application. The vast majority of ophthalmic procedures are performed using topical anesthesia. Minimizing the use of needles and invasive techniques to deliver anesthesia has been an ongoing trend in ophthalmic anesthesia over the past decade.

Recent practices for ocular anesthesia involving an intravitreal injection include subconjunctival injection of $2 \%$ aqueous lidocaine, topical $2 \%$ lidocaine drops and topical $0.5 \%$ tetracaine. The technique for $2 \%$ subconjunctival lidocaine has been described in a multicenter, randomized trial ${ }^{1}$ however, the FDA has not yet approved for this indication.

Lidocaine jelly (2\%) preparations have been reported to be beneficial in upper airway, ${ }^{2,3}$ dental, ${ }^{4}$ urogenital, ${ }^{5}$ and gastrointestinal procedures. ${ }^{6}$ The use of lidocaine topical anesthetic as a single agent for ocular procedures has been reviewed in several studies. ${ }^{7-13}$ Although considered an off-label use of lidocaine $2 \%$ (urogenital) jelly, many of these ophthalmic studies had positive findings. It has been theorized, and recent studies support the idea, that gel formulations of lidocaine may enhance anesthetic effect, and therefore, be superior to anesthetic solutions for topical cataract surgery. ${ }^{7-10}$ The viscous nature of gel formulations is thought to lengthen contact time with pain-sensitive ocular structures, resulting in better anesthesia at lower drug concentrations. In fact, a pilot study demonstrated superior anesthetic efficacy of adjunctive lidocaine gel with retrobulbar anesthesia for 25-gauge vitrectomy.

Several studies, dating back to the 1970s, suggest that lidocaine is bactericidal and bacteriostatic, and may supplement in preventing and treating surgical site infections. More recently, Parr et al demonstrated that clinical doses of lidocaine in surgical site infections inhibited the growth of Escherichia faecalis, Escherichia coli, Pseudomonas aeruginosa, Staphylococcus aureus, methicillin-resistant S. aureus (MSRA), and vancomycin-resistant enterococci in a dose-dependent fashion. ${ }^{14}$ In a guinea pig model, Stratford et al evaluated the benefit of lidocaine on bacterial colonization of surgical wounds by comparing two wounds on each animal. One was infiltrated with lidocaine $2 \%$, and the other left untreated. Results showed a $>70 \%$ decrease in colony counts in the wound treated with lidocaine. ${ }^{15}$ Overall, lidocaine has been proven to be effective against Candida albicans, E. coli, E. faecalis, Haemophilus influenzae, MRSA, S. aureus, Staphylococcus epidermidis, P. aeruginosa, Streptococcus pneumoniae. Concentrations of lidocaine that are greater than $2 \%$ (and up to $5 \%$ ) can be effective in stopping bacterial growth. ${ }^{16-18}$
The need for safe, long-acting topical ocular anesthetic agents is an ongoing need for ophthalmology practices. This has been highlighted with the adoption of intravitreal administration of pharmacologic agents for many retina vascular diseases. In short, there has been an explosion in the number of intravitreal injections in the past few years, with some one million injections being performed annually. Intravitreal injections are done in the office setting and are most commonly done with topical anesthesia. Other retinal procedures requiring longer-acting topical anesthetic agent include panretinal photocoagulation, focal laser coagulation, laser retinopexy in the treatment of retinal tears, cryotherapy of retinal tears and detachments, and vitreoretinal surgery. Many surgical procedures are also done using topical anesthetic agents, including cataract surgery, glaucoma filtration surgery, anterior segment lasers, and strabismus surgery.

Herein we describe the lidocaine 3.5\% gel (Akten ${ }^{\mathrm{TM}}$; Akorn, Buffalo Grove, IIlinois, USA) that was FDA-approved for all ophthalmic procedures in October 2008. This gel is a preservative-free lidocaine-based anesthetic gel consisting of $35 \mathrm{mg} / \mathrm{mL}$ of lidocaine hydrochloride. The preparation is $\mathrm{pH}$ neutral. The gel contains hydroxypropylmethyl cellulose, allowing for extended corneal and conjunctival contact, which has been demonstrated to provide effective topical anesthesia. Furthermore, the viscous solution likely preserves exposed epithelial surfaces. Akten also has the benefit of being 50\% less viscous compared to non-ophthalmic gel allowing for drop application. These properties of Akten impart numerous advantages in its use for ophthalmic procedures. The reduced viscosity of lidocaine $3.5 \%$ gel, relative to lidocaine $2 \%$, also allows for the preparation to be easily washed off of the eye so that debris and bacteria do not remain trapped beneath the viscous vehicle. This provides a potential advantage against intraocular infection that was not possible with the lidocaine $2 \%$ off-label jelly. ${ }^{19}$ The lower viscosity lidocaine $3.5 \%$ gel also maintains a homogeneous, regular surface allowing for unimpaired observation of anterior segment and retinal structures, so important for performing intraocular procedures. These properties of Akten 3.5\% gel have been shown to result in less epithelial irregularities and toxicity than topical anesthetic solutions that do not contain hydroxypropylcellulose..$^{10,20}$

\section{Chemical structure}

The active ingredient is lidocaine hydrochloride, an amino amide-type local anesthetic, first synthesized by Swedish chemist Nils Lofgren in 1943, and marketed in 1949. It is prepared by first reacting 2, 6-xylidine with chloroacetyl 


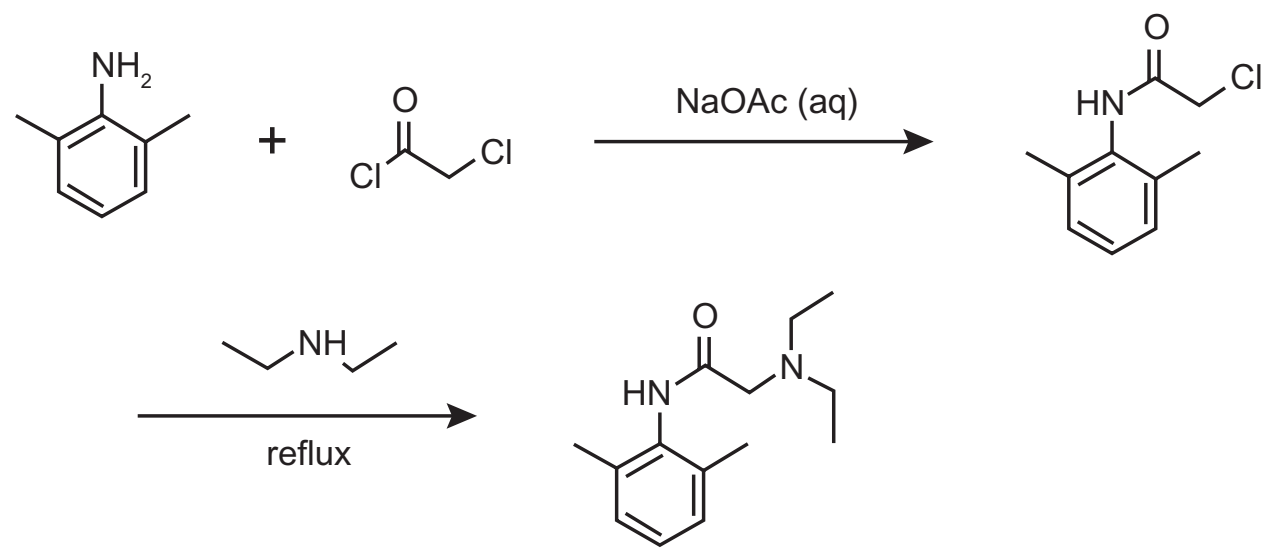

Figure I Synthesis of lidocaine.

chloride, and then by a reaction with diethylamine (see Figure 1). ${ }^{21}$ It is designated chemically as acetamide, 2-(diethylamino)-N-(2, 6-dimethylphenyl) monohydrochloride with a molecular formula of $\mathrm{C} 14 \mathrm{H} 22 \mathrm{~N} 2 \mathrm{O} \mathrm{HCl}$ and molecular weight of 270.8 .

Lidocaine hydrochloride acts by stabilizing the neuronal membrane by blocking the fast voltage gated sodium $\left(\mathrm{Na}^{+}\right)$ channels, preventing the postsynaptic neuron from depolarizing. This affects local anesthetic action by failing to transmit an action potential. ${ }^{22}$

The plasma binding of lidocaine is dependent on drug concentration and the fraction bound is inversely proportional to the concentration. At concentrations of 1 to $4 \mu \mathrm{g} / \mathrm{mL}$ of free base, $60 \%$ to $80 \%$ of lidocaine is protein bound. Lidocaine is $90 \%$ metabolized in the liver.

\section{Indication/rationale/evidence of support use}

A pivotal prospective, randomized, double-blinded, multicenter Phase III clinical trial demonstrated the efficacy of lidocaine $3.5 \%$ gel. This study led to FDA-approval of Akten in 2008. Eight study centers participated in this trial. A total of 209 subjects were enrolled with 54, 51, 53, and 51 subjects randomized to the sham, lidocaine $1.5 \%$ gel, lidocaine $2.5 \%$ gel, lidocaine $3.5 \%$ gel groups, respectively. Patients were evaluated for efficacy and safety of all of the lidocaine gel preparations. Anesthesia was determined to be present if there was an absence of pain after pinching the conjunctiva with $0.3-\mathrm{mm}$ forceps. Anesthesia was achieved within 5 minutes of application in $92 \%$ of the subjects. Of this group, $87 \%$ achieved anesthesia within 1 minute. The mean time to anesthesia onset was not affected by the dose. Anesthesia generally occurred between 20 seconds to 1 minute and persisted for 5 to 30 minutes. The mean time to anesthesia onset in the clinical study was 60 seconds, with a median onset time of 40 seconds. The duration of anesthesia ranged from 5 minutes to 40 minutes, with mean anesthesia duration of 13.4 minutes. Akten 3.5\% provided a longer duration of approximately 15 minutes. ${ }^{20}$

Page et al recently evaluated the use of lidocaine hydrochloride ophthalmic gel in a literature review of 25 studies, including 15 prospective randomized controlled trials (RCT), ${ }^{7,8,11,20,23-33} 6$ nonrandomized prospective studies, 2 animal studies, 1 microbiologic study, and 2 letters to the editor. Data from the 15 prospective RCTs are summarized in Table $1 .{ }^{34}$ Two of these were double-blinded, of which one was the previously described Akten clinical trial., ${ }^{7,20}$ The 15 RCTs included a total of 933 patients. Five of the 13 RCTs, including the randomized trial of Akten, revealed a statistically significantly lower pain score in the lidocaine gel group compared with other modalities of ocular anesthesia. Five RCTs compared the requirement for additional anesthetic applications for patient comfort, ${ }^{8,11,23,24,32}$ and 4 of these demonstrated that lidocaine gel resulted in a statistically significantly lower number of supplemental anesthetic applications. . $^{81,23,24}$ Two papers found significantly higher intracameral lidocaine levels in those receiving lidocaine gel than lidocaine drops. ${ }^{7,12}$ In this review of ocular anesthesia, only the Akten trial described time to onset and duration of anesthesia in detail. ${ }^{20}$ Several studies evaluated surgeon and patient satisfaction. Soliman et al reported that $93.3 \%$ of patients reported satisfaction undergoing cataract surgery with lidocaine jelly versus $83.3 \%$ of those who received bupivicaine drops. ${ }^{8}$ In an another small study, Segev et al reported that 14 of 15 patients preferred the lidocaine jelly over previous experience of retrobulbar injection during penetrating keratoplasty. ${ }^{35}$ In conclusion, the series of papers compared in the usage of lidocaine gel suggests it is often 


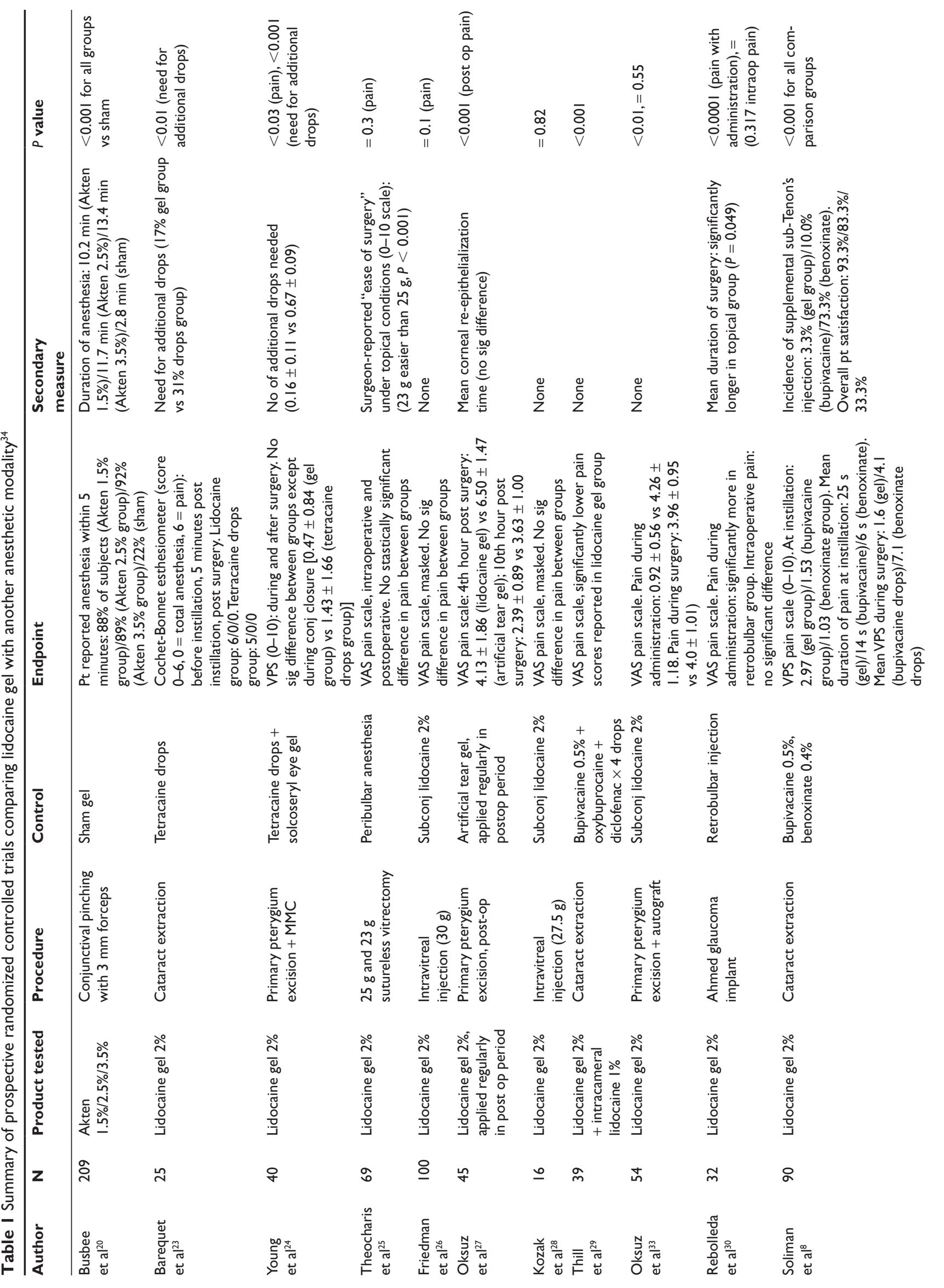




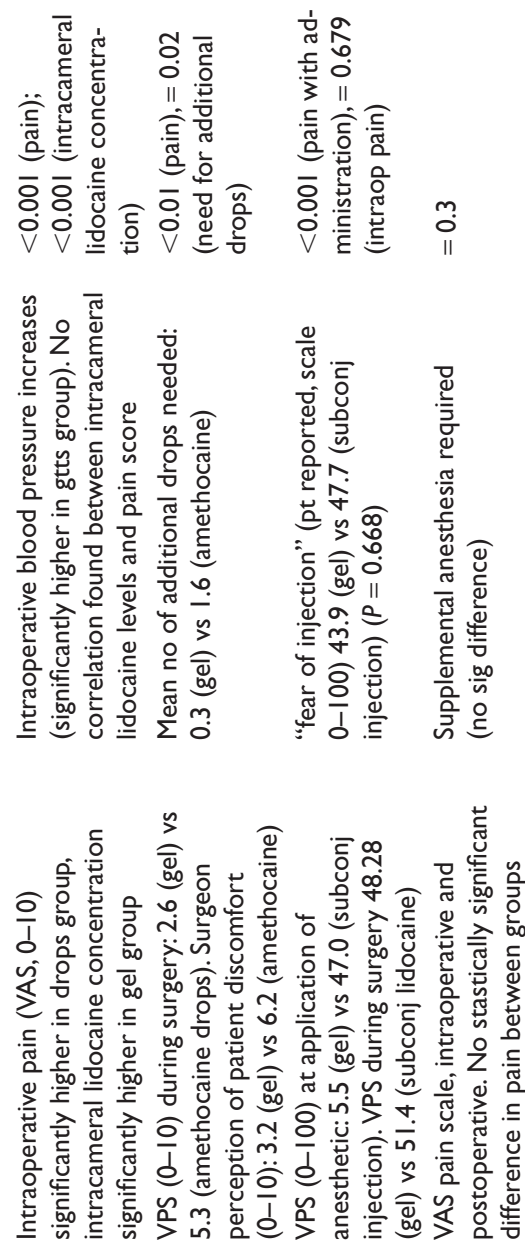

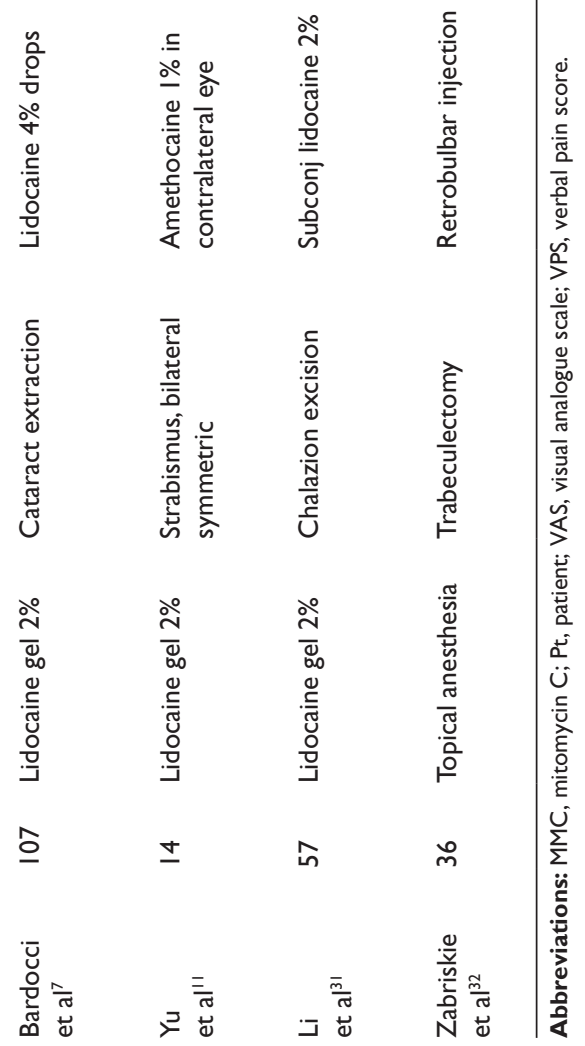

more effective than other anesthesia modalities in the prevention of procedure-related ocular pain.

The dosing strategy for Akten has been described and tailored for the use in intravitreal injections. The following dosing strategy maximizes anesthesia while providing appropriate antiseptic technique. One drop of lidocaine $3.5 \%$ gel is instilled on the ocular surface. After 2 to 3 minutes betadine solution is used to clean the ocular surface. Another drop of lidocaine $3.5 \%$ gel is then reapplied and allowed to sit on the ocular surface for at least one additional minute. Typically the patient is asked to gently close their eyes during the reapplication. Akten may be reapplied to maintain anesthetic effect. Prior to the intravitreal injection, betadine is reapplied in the area of intended injection.

Akten can be used for procedures that require an extended treatment time. Due to its viscous formulation, systemic absorption through the nasolacrimal system should be reduced, therefore reducing the potential for systemic toxicity.

Procedures that may require more extensive use of topical anesthesia include cataract surgery, trabeculectomy, pars plana vitrectomy, refractive surgery, and suture adjustment after strabismus surgery.

To date approximately 1 million patients have received topical anesthesia using Akten gel. Application of lidocaine $3.5 \%$ gel as described above typically results in a pain-free or minimally painful ocular procedure. Prior to the advent, of Akten, topical anesthetic agents included subconjunctival lidocaine and anesthetic-soaked pledgettes for intravitreal injections, and tetracaine preparations. In our experience, patients commonly note a difference in comfort between the currently used lidocaine $3.5 \% \mathrm{gel}$ and other previously administered topical anesthetic agents. Another benefit of Akten is the vast majority of patients who have anesthesia done with Akten gel do not suffer the corneal epithelial and surface irregularities that typically occur due to the toxic nature of other anesthetic preparations. This eases the post-operative period and reduces the need for artifical tear lubrication and in our experience use of Akten has replaced the need for additional intracameral lidocaine in patients undergoing topical cataract surgery. It is hypothesized that ocular penetration of lidocaine $3.5 \%$ gel results in high anterior chamber levels, adequately anesthetizing the iris and ciliary body, preventing intraoperative discomfort. Finally, we have observed a significant reduction in intraoperative pain in patients undergoing pars plana vitrectomy. Although a retrobulbar block is the primary modality for anesthesia, Akten gel applied preoperatively has been demonstrated to significantly 
reduce the potential discomfort at the beginning of a pars plana vitrectomy.

\section{Adverse effects}

The adverse events demonstrated by the Akten trial revealed an adverse event rate ranging from $2 \%$ to $6 \%$ across all treatment groups. ${ }^{21}$ The most common adverse event was corneal staining. Corneal staining was reported by $6 \%$ (3 patients) in the lidocaine 3.5\% gel group, and $2 \%$ (1 patient) in the sham group. All patients showed resolution of their corneal epithelial changes within 24 hours. Other common adverse events include conjunctival hyperemia (6\%) and conjunctival hemorrhage $(3 \%)$. Both of these findings are likely due to the technique $(0.3$ forceps pinching of conjunctiva) by which pain was evaluated in the study. No serious adverse events were noted.

\section{Precautions}

With regard to use in pregnancy, reproduction studies for lidocaine have been performed in both rats and rabbits. There is no evidence of harm to the fetus at subcutaneous doses up to $50 \mathrm{mg} / \mathrm{kg}$ lidocaine in the rat model. This dosage is more than 800 -fold greater than the human dose on a body weight basis. There are no well- controlled studies in pregnant women.

In terms of use in nursing mothers, lidocaine is secreted in human milk. The clinical significance of this is unknown. Caution should be exercised when lidocaine preparations are administered to nursing women.

In the pediatric population, lidocaine safety and efficacy has been extrapolated from studies in older subjects and studies in pediatric patients using different formulations of lidocaine.

Prolonged use of a topical ocular anesthetic may produce permanent corneal opacification and ulceration due to delayed wound healing. Topical ocular application of lidocaine $3.5 \%$ gel is not expected to result in systemic exposure.

\section{Conclusion}

Akten gel, a preservative-free, $\mathrm{pH}$ neutral, 3.5\% lidocainebased anesthetic gel, appears to have significant benefits over standard topical ocular anesthetic agents. Its viscous solution allows for prolonged contact time and, therefore, may provide superior anesthesia relative to other topical solutions. Higher dose lidocaine may also protect from bacterial infections and could potentially reduce the risk of intraocular infections relative to other topical anesthetic agents. ${ }^{16-18}$ The hydroxypropylcellulose aids in preserving the corneal epithelium after topical anesthesia is applied, serving an added benefit in the aged population in whom most of the ophthalmic procedures are done. With the dramatic rise in intravitreal injections and other ophthalmology procedures comes a need for better topical anesthetic agents. It is believed Akten gel provides superior anesthesia, while minimizing side effects.

\section{Disclosure}

Drs Reichel and Busbee have a commercial interest (royalties) in Akten.

\section{References}

1. Rosenfeld PJ, Brown DM, Heier JS, Boyer DS; MARINA Study Group. Ranibizumab for neovascular age-related macular degeneration. $N$ Engl J Med. 2006;355(14):1419-1431.

2. Zainudin BM, Rafia MH, Sufarlan AW. Topical nasal anesthesia for fibreoptic bronchoscopy: lidocaine spray or gel? Singapore Med J. 1993;34(2):148-149.

3. Chan ST, Them CS. The effects of $2 \%$ lidocaine gel on incidence of retching with the use of the laryngeal mask airway. Anaesthesia. 1995;50(3): 257-258.

4. Donaldson D, Gelskey SC, Landry RG, Mathews DC, et al. A placebocontrolled multi-centered evaluation of an anaesthetic gel (Oraqix) for periodontal therapy. J Clin Periodontol. 2003;30(3):171-175.

5. Kalafi H, Cengiz M, Demir N. Intrauterine lidocaine gel application for pain relief during and after hysterosalpingography. Int J Gynaecol Obstet. 2003;83(1):65-67.

6. Mallick S, Humbert M, Braud F, Fofana M, et al. Local anesthesia before transrectal ultrasound guided prostate biopsy: comparison of 2 methods in a prospective, randomized clinical trial. J Urol. 2004;171:730-733.

7. Bardocci A, Lofoco G, Perdicaro S, Ciucci F, et al. Lidocaine 2\% gel versus lidocaine 4\% unpreserved drops for topical anesthesia in cataract surgery: a randomized controlled trial. Ophthalmology. 2003;110(1):144-149.

8. Soliman MM, Macky TA, Samir MK. Comparative clinical trial of topical anesthetic agents in cataract surgery: lidocaine $2 \%$ gel, bupivacaine $0.5 \%$ drops, and benoxinate $0.4 \%$ drops. $J$ Cataract Refract Surg. 2004;30(8):1716-1720.

9. Gaynes BI. Lidocaine gel versus drops. Ophthalmology. 2003;110(12):2429-2430.

10. Irle S, Luckefahr MH, Tho Seeth T. Tetracaine drops versus lidocaine gel for topical anaesthesia in cataract surgery. Klin Monatsbl Augenheillkd. 2003;220(9):625-628.

11. Yu CB, Wong VW, Fan DS, Yip WW, et al. Comparison of lidocaine $2 \%$ gel versus amethocaine as the as the sole anesthetic agent for strabismus surgery. Ophthalmology. 2003;110(7):1426-1429.

12. Kwok AK, Young AL, Lam DS. Efficacy of lidocaine gel for outpatient laser treatment in inflamed eyes. Eye. 2001;15:608-611.

13. Assia EI, Pras E, Yehezkel M, Rotenstreich Y, et al. Topical anesthesia using lidocaine gel for cataract surgery. J Cataract Refract Surg. 1999;25(5):635-639.

14. Parr AM, Zoutman DE, Davidson JS. Antimicrobial activity of lidocaine against bacteria associated with nosocomial would infection. Ann Plast Surg. 1999;43:239-245.

15. Stratford AF, Zoutman DE, Davidson JS. Effect of lidocaine and epinephrine on Staphylococcus aureus in a guinea pig model of surgical wound infection. Plast Reconstr Surg. 2002;110:1275-1279.

16. Noda H, Saionji K, Miyazaki T. Antibacterial activity of local anesthetics. Masui. 1990;39:994-1001. 
17. Sakuragi T, Ishino H, Dan K. Bactericidal activity of clinically used local anesthetics on Staphylococcus aureus. Reg Anesth. 1996;21: 239-242.

18. Aydin ON, Eyigor M, Aydin N. Antimicrobial activity of ropivacaine and other local anaesthetics. Eur J Anaesthesiol. 2001;18:687-694.

19. Miller JJ, Scott IU, Flynn HW Jr, Smiddy WE, et al. Endophthalmitis after cataract surgery (2000-2004): incidence, clinical settings, and visual acuity outcomes after treatment. Am J Ophthalmol. 2005; 139(6):983-987.

20. Busbee BG, Alam A, Reichel E. Lidocaine hydrochloride gel for ocular anesthesia: results of a prospective, randomized study. Ophthalmic Surg Lasers Imaging. 2008;39(5):386-390.

21. Reilly TJ. The preparation of lidocaine. J Chem Ed. 1999:76(11):557.

22. Catterall WA. Molecular mechanisms of gating and drug block of sodium channels. Novartis Found Symp. 2002;241:206-218.

23. Barequet IS, Soriano ES, Green WR, O'Brien TP. Provision of anesthesia with a single application of lidocaine $2 \%$ gel. J Cataract Refract Surg. 1999;25(5):626-631.

24. Young AL, Leung GY, Cheng LL, Lau TT, et al. Randomised controlled trial on the effectiveness of lidocaine gel vs tetracaine drops as the sole topical anaesthetic agent for primary pterygium surgery. Eye. 2009;23(7):1518-1523.

25. Theocharis IP, Alexandridou A, Tomic Z. A two-year prospective study comparing lidocaine $2 \%$ jelly versus peribulbar anaesthesia for $25 \mathrm{G}$ and $23 \mathrm{G}$ sutureless vitrectomy. Graefes Arch Clin Exp Ophthalmol. 2007;245(9):1253-1258.
26. Friedman SM, Margo CE. Topical gel vs subconjunctival lidocaine for intravitreous injection: a randomized clinical trial. Am J Ophthalmol. 2006;142(5):887-888.

27. Oksuz H, Tamer C. Pain relief after pterygium surgery with viscous lidocaine. Ophthalmologica. 2006;220(5):323-326.

28. Kozak I, Cheng L, Freeman WR. Lidocaine gel anesthesia for intravitreal drug administration. Retina. 2005;25(8):994-998.

29. Thill M, Zeitz O, Richard I, Richard G. Lidocaine gel versus combined topical anesthesia using bupivicaine, oxybuprocaine, and diclofenac eyedrops in cataract surgery. Ophthalmologica. 2005;219(3):167-170.

30. Rebolleda G, Munoz-Negrete FJ, Gutierrez-Ortiz C. Topical plus intracameral lidocaine versus retrobulbar anesthesia in phacotrabeculectomy: prospective randomized study. J Cataract Refract Surg. 2001;27(8):1214-1220.

31. Li RT, Lai JS, Ng JS, Law RW, et al. Efficacy of lignocaine $2 \%$ gel in chalazion surgery. Br J Ophthalmol. 2003;87(2):157-159.

32. Zabriskie NA, Ahmed II, Crandall AS, Daines B, et al. A comparison of topical and retrobulbar anesthesia for trabeculectomy. J Glaucoma. 2002;11(4):306-314.

33. Oksuz H, Tamer C. Efficacy of lidocaine $2 \%$ gel in pterygium surgery. Acta Ophthalmol Scand. 2005;83(2):206-209.

34. Page MA, Fraunfelder FW. Safety, efficicay, and patient acceptability of lidocaine hydrochloride ophthalmic gel as a topical ocular anesthetic for use in ophthalmic procedures. Clin Ophthalmol. 2009;3:601-609.

35. Segev F, Voineskos AN, Hui G, et al. Combined topical and intracamera anesthesia in penetrating keratoplasty. Cornea. 2004;23(4):372-376.
Local and Regional Anesthesia

\section{Publish your work in this journal}

Local and Regional Anesthesia is an international, peer-reviewed, open access journal publishing on the development, pharmacology, delivery and targeting and clinical use of local and regional anesthetics and analgesics. The journal welcomes submitted papers covering original research, basic science, clinical studies, reviews \& evaluations,

\section{Dovepress}

guidelines, expert opinion and commentary, case reports and extended reports. The manuscript management system is completely online and includes a very quick and fair peer-review system, which is all easy to use. Visit http://www.dovepress.com/testimonials.php to read real quotes from published authors. 